\title{
Frequency Domain Interleaving for Dense WDM Passive Optical Network
}

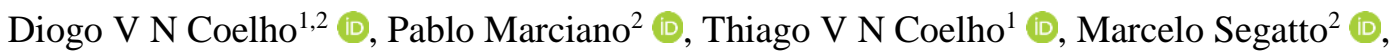 \\ Maria Jose Pontes ${ }^{2}$ \\ ${ }^{1}$ Universidade Federal de Juiz de Fora, Juiz de Fora, MG, Brazil, 36036-330, diogo.coelho@ufjf.edu.br \\ ${ }^{2}$ Universidade Federal do Espírito Santo,Vitória, ES, Brazil,29075-910, pablorafael.mcx@gmail.com, \\ thiago.coelho@ufjf.edu.br,marcelo.segatto@gmail.com,mjpontes@ele.ufes.br
}

\begin{abstract}
In this work, we introduce a new concept of frequency interleaving applied to passive optical networks based in Dense Wavelength Division Multiplexing (DWDM). In order to explore colorless ONUs approach, a single and centralized optical source is used to create the interleaved wavelengths for the downstream and upstream path. In this case, frequency comb generation implemented by recirculating frequency shifting technique allowed the creation of all the wavelengths used in each path. The reception operation is accomplished by introducing, at the ONU side, a combination of a set of optical passive elements. The system with 4 $\times 12.5 \mathrm{Gbit} / \mathrm{s}$ NRZ-OOK (Not Return to Zero - On-Off Keying) modulation format were simulated through $20 \mathrm{~km}$ of standard single mode fiber link in both transmission paths. In order to guarantee a high quality optical frequency comb source, the optical carrier-to-noise-ratio (OCNR) was evaluated as well as BER (bit error rate), to analyze the system performance. All devices models and simulations were performed in Matlab environment.
\end{abstract}

Index Terms - DWDM, PON, spectral-efficiency, frequency interleaving.

\section{INTRODUCTION}

Wavelength division multiplexed passive optical networks (WDM-PONs) have been extensively studied for applications that involve large bandwidth over the last years. This strong growth observed in WDM-PONs is mainly due to the communication services demand from end-users such as residential and business customers. Applying WDM-PONs to solve this growing demand for bandwidth can contribute to lower deployment costs and strongly disseminate their use.

The nonexistence of active devices connecting the Optical Line Terminal (OLT) to the Optical Network Units (ONUs) is one of the advantages of using PONs. It means enhancing end-to-end transparency, diminishing hardware processing and, consequently, preventing effects produced by electrical noise sources. Furthermore, the high bandwidth combined with the infrastructure, which can be shared among end-users, reduces the costs and simplify the maintenance as well as the operation [1].

On the other hand, when it comes to WDM networks, issues related to wavelength assignment should be considered. Essentially, the channel bandwidth observed for traditional WDM channels 
strictly follow the ITU-T wavelength grids and spacing, e.g. $50 \mathrm{GHz}, 100 \mathrm{GHz}$. Moreover, in the context of WDM-PONs the concept of spectrum efficiency should be incorporated. It requires optimized channel allocation and related improvements such as elastic optical networks (EONs) with elevated granularity (DWDM with $12.5 \mathrm{GHz}$ frequency spacing) [2].

However, the need for a wavelength region for downstream and another one for upstream represents an inefficient use of the available spectrum. We should consider a future access system design assuming that existing PONs should coexist [3-4]. Theses features will allow progressive migration of existing subscribers as they move to the new technology, without disrupting services for customers on the legacy PON [4]. In order to improve the spectral efficiency and provide coexistence with previous generations of PONs, the frequency interleaving of downstream and upstream signals could be considered as a solution for the wavelength plan of the next generation of optical access networks.

In this work, high-capacity optical DWDM access networks based on the concept of frequency interleaving is introduced and numerically simulated, as an efficient method to use the available spectrum and provide coexistence with legacy PON standards, since the downstream and upstream wavelengths are closer to each other and share the same frequency band. All the devices models were created and simulated in Matlab. The methodology used in this work was similar the one utilized by the authors in [5] that created several optical device models for backbone network planning simulations.

The paper is organized as follow. Section II explains the concept of frequency interleaving for DWDM access networks. Section III describes the principle of the optical multi-carrier generator used in this work. Sections IV and V describes, respectively, the demultiplex operation and architecture for optical access networks with frequency interleaving. Section VI presents the simulations discussion and the results. Finally, the conclusions are given in Section VII.

\section{FREQUENCY DOMAIN INTERLEAVING CONCEPT}

A single and centralized optical comb source is responsible to generate multiple equal spaced and frequency locked wavelength channels, which will be modulated, with a symbol rate equal the frequency space between the wavelengths, for downstream and upstream transmission. Due to the nature of the comb source, any oscillation in the laser seed power will be transfer to all useful wavelengths at the same proportion; i.e., the only power difference between the wavelengths will be defined by the flatness of the comb source. The even wavelengths generated will be used in the downstream path, while the odd ones in the upstream path, creating an interleaving pattern between consecutive wavelengths.

The demultiplexing operation is introduced at the ONU side and is processed by the use of an alloptical passive element (Mach-Zehnder interferometer - MZI). For practical purposes, this processing 
can be implemented with silicon-on-insulator (SOI) technology in photonic integrated circuits (PICs) [6-7]. In its most basic form, the technology allows to implement passive optical components, such as splitters, filters, (de)multiplexers, polarization handling components, interferometers, resonators and their combination with coupling structures to optical fibers or to free space optical elements [7].

In this concept, four wavelengths will be used in the downstream and another four in the upstream path. In this context, each dynamic group of 64 ONUs will share one of the four wavelengths in time domain.

\section{RECIRCULATING FREQUENCY SHIFTING (RFS)}

Multitudes of methods are reported on the literature that allows generating a series of discrete, equally spaced frequency lines. However, for optical communications and RF (radio-frequency) photonics applications, techniques using opto-electronic devices, such as optical modulators, are suitable due spectral flatness, robustness and tunability [8]. A technique based on ultra-dense and parametric comb is described in [9] that generates spectral lines spaced by $6.25 \mathrm{GHz}$ and $3 \mathrm{~dB}$ spectral flatness. Although this technique creates a huge number of carrier lines within $100 \mathrm{~nm}$ of optical bandwidth ( $\mathrm{C}+\mathrm{L}$ band), the setup is quite intricate that requires elements such as dispersion flattened highly nonlinear fibers and high CW laser powers. A method based on a gain-switched comb, which produces a $12.5 \mathrm{GHz}$ spaced channels over the C-band is reported in [10]. The experimental setup is practical, but this technique generates a limited number of frequency lines (six comb lines) with $3 \mathrm{~dB}$ flatness and requires a high RF sinusoidal power (around $24 \mathrm{dBm}$ ) in the Fabry-Perot laser diode used as slave. Nevertheless, an interesting method, that uses cascaded intensity and dual-parallel modulators, is presented in [11]. In this case, however, the complexity increases with the number of generated optical carriers, since they require high RF frequency and power control of each optical modulator [11].

The RFS technique, as sketch in Fig. 1, presents very good stability, flatness, flexibility on wavelength spacing control and low driving voltages [12]. Although this optical multi-carrier generator presents a relatively complex configuration, it is an effective tool as an optical multi-carrier source and can generate hundreds of them using commercially available opto-electronic devices. For this reason, RFS technique was chosen as the optical comb source implemented in our proposal.

In the RFS technique, a continuous wave (CW) laser is utilized as a seed, which determines the starting wavelength of the generated optical multi-carriers set. The optical amplifier (OA) in the ring, as illustrated in Fig. 1, compensates any transmission loss observed in the ring as well as the insertion loss in the coupler and optical modulator (IQ-MZM). An optical band pass filter (OF) defines the wavelength range of the optical subcarriers generated.

Every time the optical signal passes through the complex modulator (IQ-MZM), which is adjusted to generate a single side-band suppressed carrier optical signal (SSB-SC), the frequency of the signal 
will be shifted according to the frequency $\left(f_{c}\right)$ of the sine wave input. After each round trip, the input $\mathrm{CW}$ will occupy the previous empty position. As a result, new optical subcarriers will be created on each round trip in the ring. The frequency of the IQ-MZM sine wave defines the spacing between the optical subcarriers.
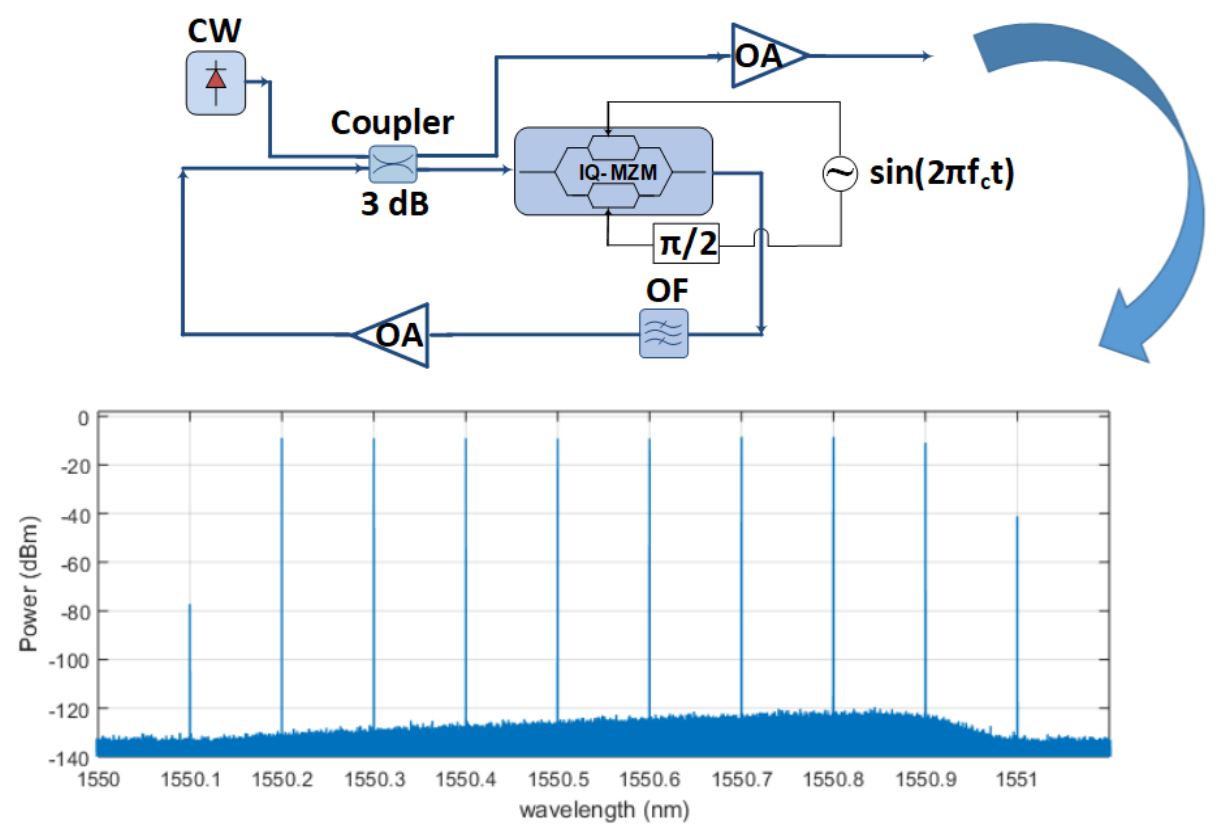

Fig. 1. Recirculating Frequency Shifting scheme.

Figure 1 shows the result of the RFS model obtained by simulation. As aforementioned all the devices models and simulations, used in this work, where performed in Matlab. The CW laser model emits in $1550.2 \mathrm{~nm}$ with $10 \mathrm{dBm}$ of optical power, $40 \mathrm{~dB}$ of optical carrier-to-noise ratio (OCNR) and has a narrow optical linewidth (>1 KHz) to guarantee low phase noise in the multiple wavelengths generated [13]. The optical amplifier (OA) model inside the ring is an erbium-doped fiber amplifier (EDFA) with $6.2 \mathrm{~dB}$ of gain and noise figure of $4.5 \mathrm{~dB}$. The amplified spontaneous emission (ASE) noise of the EDFA was also considered in the model to obtain the noise figure values as expected. The frequency $\left(\mathrm{f}_{\mathrm{c}}\right.$ ) of the RF signal is $12.5 \mathrm{GHz}$, generating optical subcarriers with $0.1 \mathrm{~nm}$ of wavelength spacing and $2.37 \mathrm{~dB}$ of flatness. The optical bandpass filter $(\mathrm{OF})$ has $0.7 \mathrm{~nm}$ of bandwidth $(\approx 87.5$ $\mathrm{GHz}$ ), which results in eight useful optical subcarriers (1550.2 $\mathrm{nm}$ to $1550.9 \mathrm{~nm})$, as shown in Fig. 1.

To guarantee a high quality optical frequency comb in high bit rate transmission, carrier-to-noise ratio (OCNR) was evaluated in terms of optical subcarrier number [13]. Fig. 2 shows the result of OCNR obtained for each subcarrier generated in the RFS model simulated.

As reported by R. Essiambre et al. [14], the OCNR needed to transmit a $12.5 \mathrm{~Gb} / \mathrm{s}$ in OOK modulation format with a bit error ratio of $10^{-3}$ is $10 \mathrm{~dB}$. As shown in Fig. 2, our frequency comb generator provides multiple wavelengths with quality that is sufficiently large for OOK transmission. 


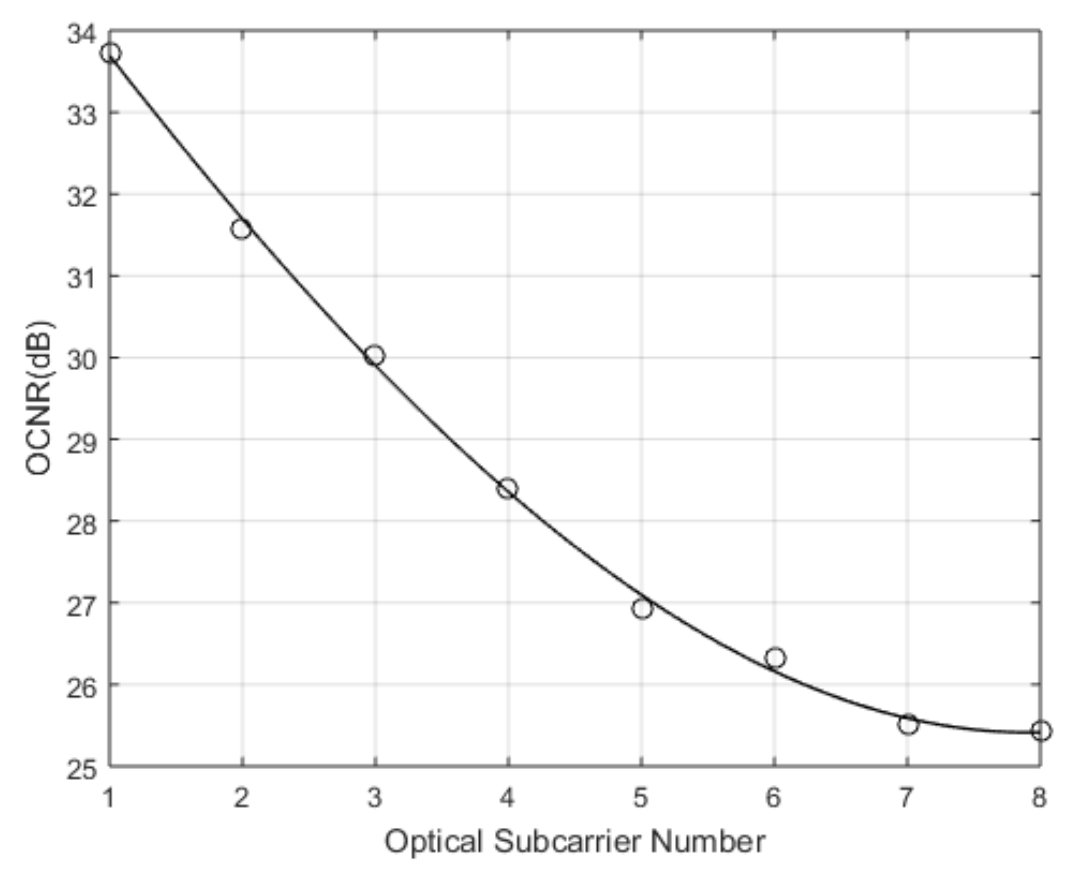

Fig. 2. Optical subcarriers generated in our simulations

\section{DEMULTIPLEX OPERATION FOR FREQUENCY INTERLEAVED DWDM-PON}

This section describes the demultiplex device for optical access networks that applies the concept of frequency interleaving, that allows downstream and upstream channels coexist in the same wavelength region. It may represents an efficient use of the available spectrum and coexistence with legacy PON systems on the same optical distribution network (ODN) [3].

The demultiplex operation, for downstream and upstream wavelengths, is based in the optical fast Fourier transform (OFFT) method [15-16]. It consists of 3-cascaded MZIs for each path with different delays $(\tau)$ and phase shifts $(\phi)$, which is illustrated in Fig. 3. The $\mathrm{T}_{\mathrm{s}}$ parameter in Fig. 3 is the symbol period used in the system transmission. The MZI device model, created in Matlab, with two inputs ( $E_{\text {in } 1}$ and $E_{\text {in } 2}$ ) and two outputs ( $E_{\text {out } 1}$ and $\left.E_{\text {out } 2}\right)$ can be mathematically defined by the transfer matrix

$$
\left[\begin{array}{l}
E_{\text {out } 1} \\
E_{\text {out } 2}
\end{array}\right]=\left[\begin{array}{cc}
\sqrt{1-k} & j \sqrt{k} \\
j \sqrt{k} & \sqrt{1-k}
\end{array}\right]\left[\begin{array}{cc}
e^{-j 2 \pi f \tau} & 0 \\
0 & e^{-j \phi}
\end{array}\right]\left[\begin{array}{cc}
\sqrt{1-k} & j \sqrt{k} \\
j \sqrt{k} & \sqrt{1-k}
\end{array}\right]\left[\begin{array}{l}
E_{i n 1} \\
E_{i n 2}
\end{array}\right],
$$

where $k$ is the coupling coefficient (0.5) of the MZI optical coupler and $f$ is the frequency vector used in the simulations [17]. 


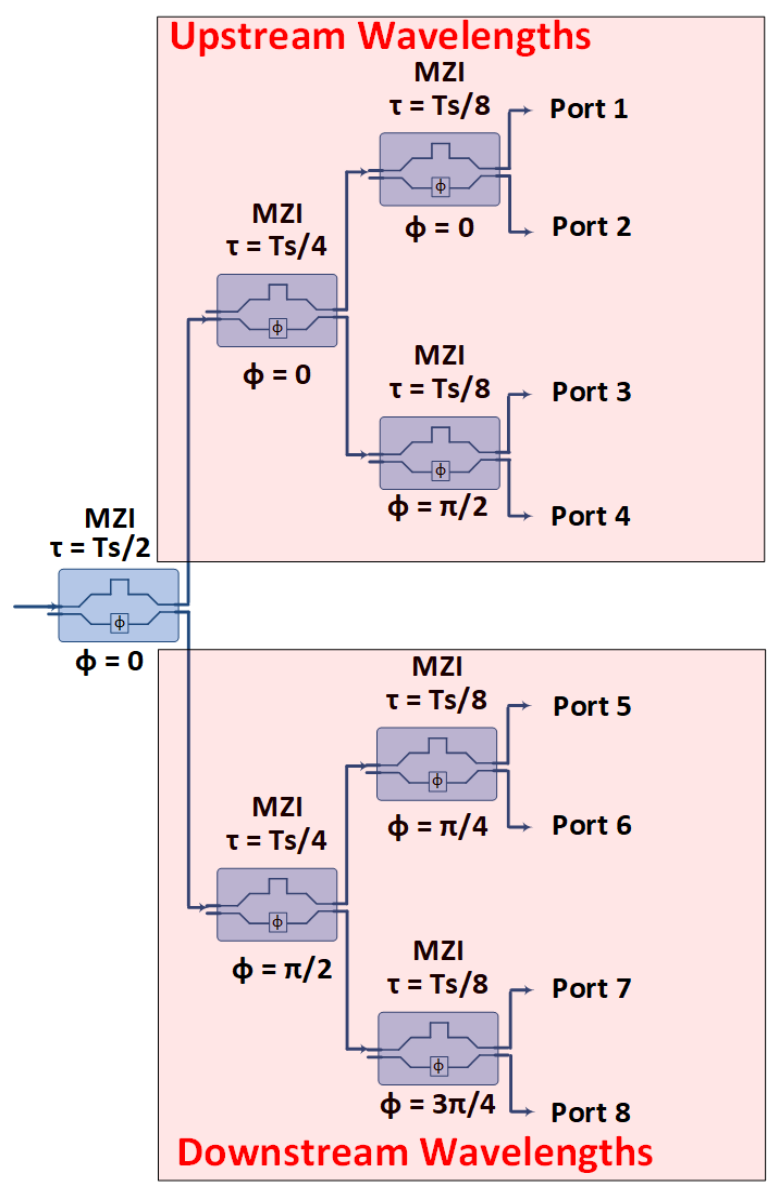

Fig. 3. MZI cascade demultiplex device.

The amplitude of the frequency response of each output port of the MZI cascade device of Fig. 3 can be obtained by applying the Fourier transform of the impulse response, in Matlab, to each port and visualized in Fig. 4. The MZI cascade acts as a multiband optical filter where each port will be responsible for extracting passively a single wavelength of the optical signal transmitted. Furthermore, the MZI cascade demultiplex device avoid $20 \mathrm{~dB}$ of crosstalk noise at the carrier frequency [18]. The symbol period used in the implementation model of the demultiplex device was 80 ps.

The advantage of this approach is that a single wavelength component of the signal can be easily extracted without the implementation of the complete structure [16]. In this case, all MZIs that are not part of the optical path to the corresponding output port can be removed, leaving only one MZI per stage [16]. Another possibility for practical implementation relies on, as aforementioned, implementation with silicon-on-insulator technology, obtaining much more compact structures [6-7]. 

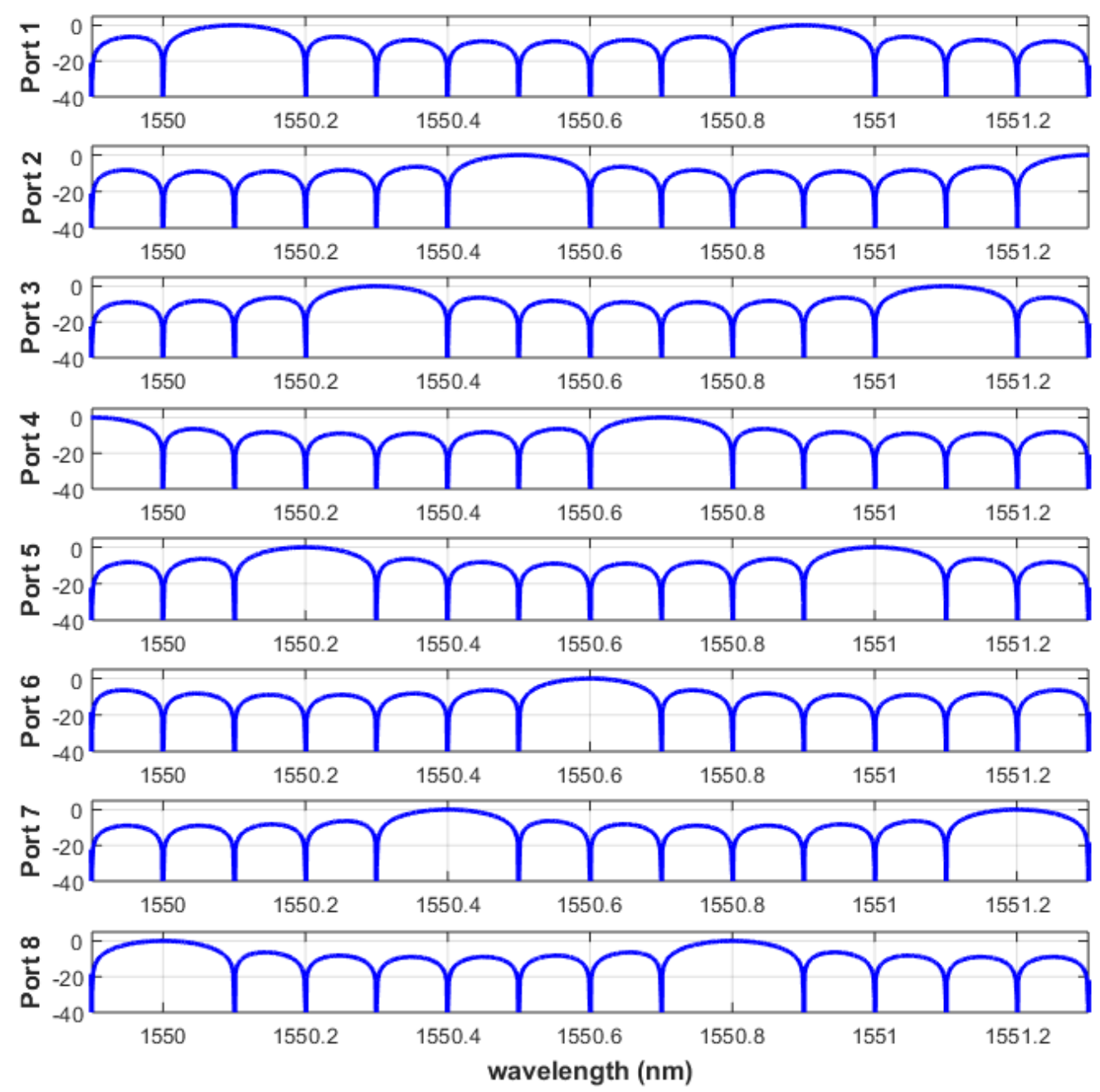

Fig. 4. MZIs cascade amplitude response.

\section{ARCHITECTURE FOR FREQUENCY INTERLEAVED DWDM-PON}

Figure 5 shows the architecture based on the concept of frequency interleaved DWDM proposed in this work. At the transmitter side, an optical multi-carrier generator creates downstream and upstream wavelengths spaced by $f_{c}$ given in $\mathrm{Hz}$ units. The multiple wavelengths, generated by the comb source, are separated by a MZI demultiplex device illustrated in Fig. 3. The odd wavelengths are modulated in NRZ-OOK modulation format, while the even wavelengths pass through the transmitter without any modulation process. After the modulation of the downstream signals, all the wavelengths (odd and even) are multiplexed by an optical combiner, creating an interleaving pattern between them.

We used a standard single mode fiber (SMF) model with attenuation factor $\alpha=0.2 \mathrm{~dB} / \mathrm{km}$, fiber core effective area $A_{\text {eff }}=80 \mu / \mathrm{m}^{2}$, fiber dispersion coefficient $\mathrm{D}=17 \mathrm{ps} / \mathrm{nm} . \mathrm{km}$ and dispersion slope $\mathrm{S}=0.09 \mathrm{ps} / \mathrm{nm}^{2} \cdot \mathrm{km}^{2}$ at $1550 \mathrm{~nm}$ wavelength. The splitter ratio used to share the optical signals among all the ONUs was 1:256, i.e., each subcarrier will be used by 64 ONUs in a TDM (Time Division 
Multiplexing) method. The thermal noise was inserted in the photodetection modeling.

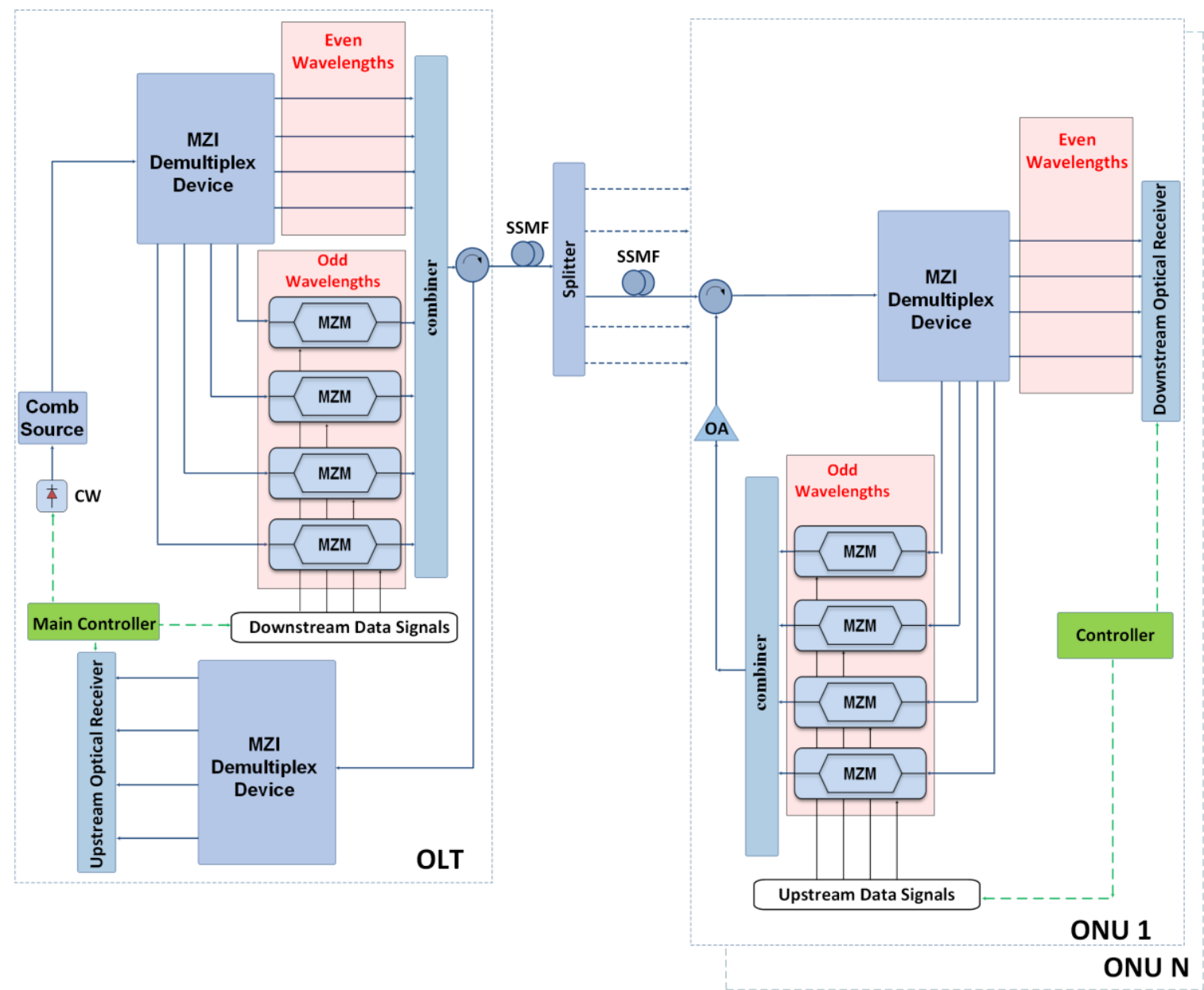

Fig. 5. Architecture scheme for frequency interleaved DWDM-PON.

At the ONU side, the MZI demultiplex device of Fig. 4 is responsible to separate the wavelengths for downstream and upstream operations. After the wavelength separation, each modulated downstream wavelength is direct-detected (DD).

In the upstream path, after the demultiplex operation, the wavelength is modulated by the upstream data, amplified and transmitted to the OLT. The main controller element in Fig. 4 performs the wavelength allocation procedure and synchronization, based on information from each ONU.

\section{Simulation AND RESUlts}

We have numerically simulated, in Matlab, the proposed architecture setup shown in Fig. 5, for downstream and upstream path.

The comb source employs the RFS technique, as previously described, to generate eight frequency locked optical subcarriers spaced by $12.5 \mathrm{GHz}(0.1 \mathrm{~nm})$. The generated subcarriers were separated, using the demultiplex device of Fig. 3, in four downstream subcarriers and four upstream subcarriers. The odd subcarriers were chosen for the downstream path and the even for upstream path, i.e., the 
upstream and downstream subcarriers are interleaved and share the same optical wavelength range. Fig. 6 shows the spectrum of the transmitted signal, where it is illustrated the modulated odd optical subcarriers (downstream) and the unmodulated even subcarriers (upstream).

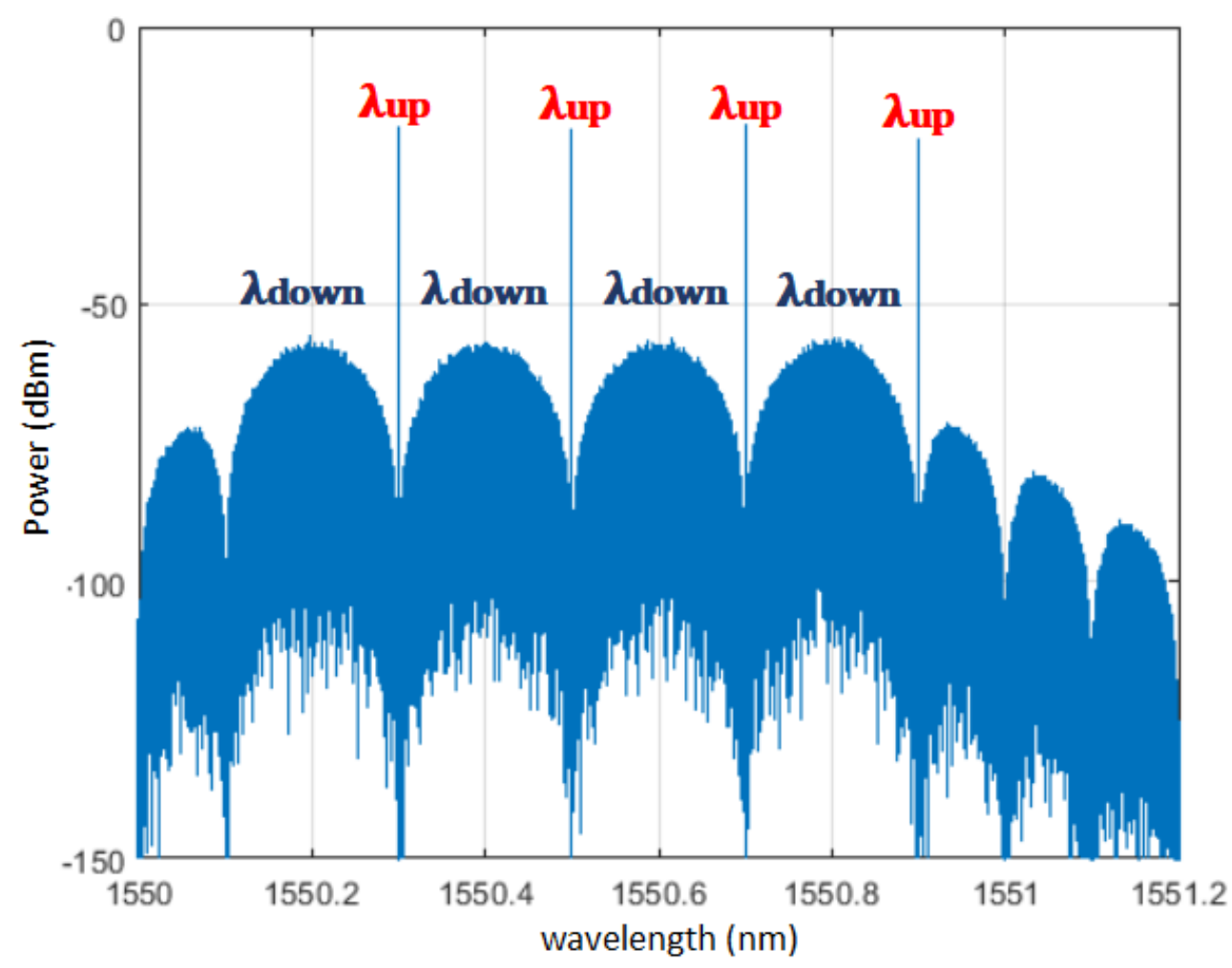

Fig. 6. Spectrum of the transmitted signal. $\lambda$ down is the modulated odd downstream optical subcarriers (wavelengths $1,3,5$ and 7). $\lambda$ up is the modulated even upstream optical subcarriers (wavelengths 2, 4, 6 and 8).

In the 4 x 12.5 Gbits/s NRZ-OOK system simulated, we used an independent pseudorandom bit sequences (PRBS) with $\left(2^{19}-1\right)$ length per wavelength. The BER curve was obtained for the ONUs maximum distance from the OLT of $20 \mathrm{~km}$ of SMF in downstream/upstream path without dispersion compensation, as can be seen in Fig. 7 and Fig. 8. The 7\% forward-error-correction (FEC) limit, around $3.8 \times 10^{-3}$, was also inserted for performance assessment.

As observed in Fig. 7 and Fig. 8, the BER performance remains under the FEC limit for received optical power higher than $-21 \mathrm{dBm}$, for the downstream, after $20 \mathrm{~km}$ of propagation in SMF. For the upstream path, the performance remains under the FEC limit for received optical power higher than $20 \mathrm{dBm}$. In Fig. 7 and Fig. 8, we observe that the wavelengths in the middle of the spectrum (wavelengths 3 and 5 for downstream; wavelengths 4 and 6 for upstream) presents worse performance in relation to the other channels. This could be explained, due the higher level of crosstalk presents in the middle wavelengths comparing with the others (wavelengths 1 and 7 for downstream; wavelengths 2 and 8 for upstream). 


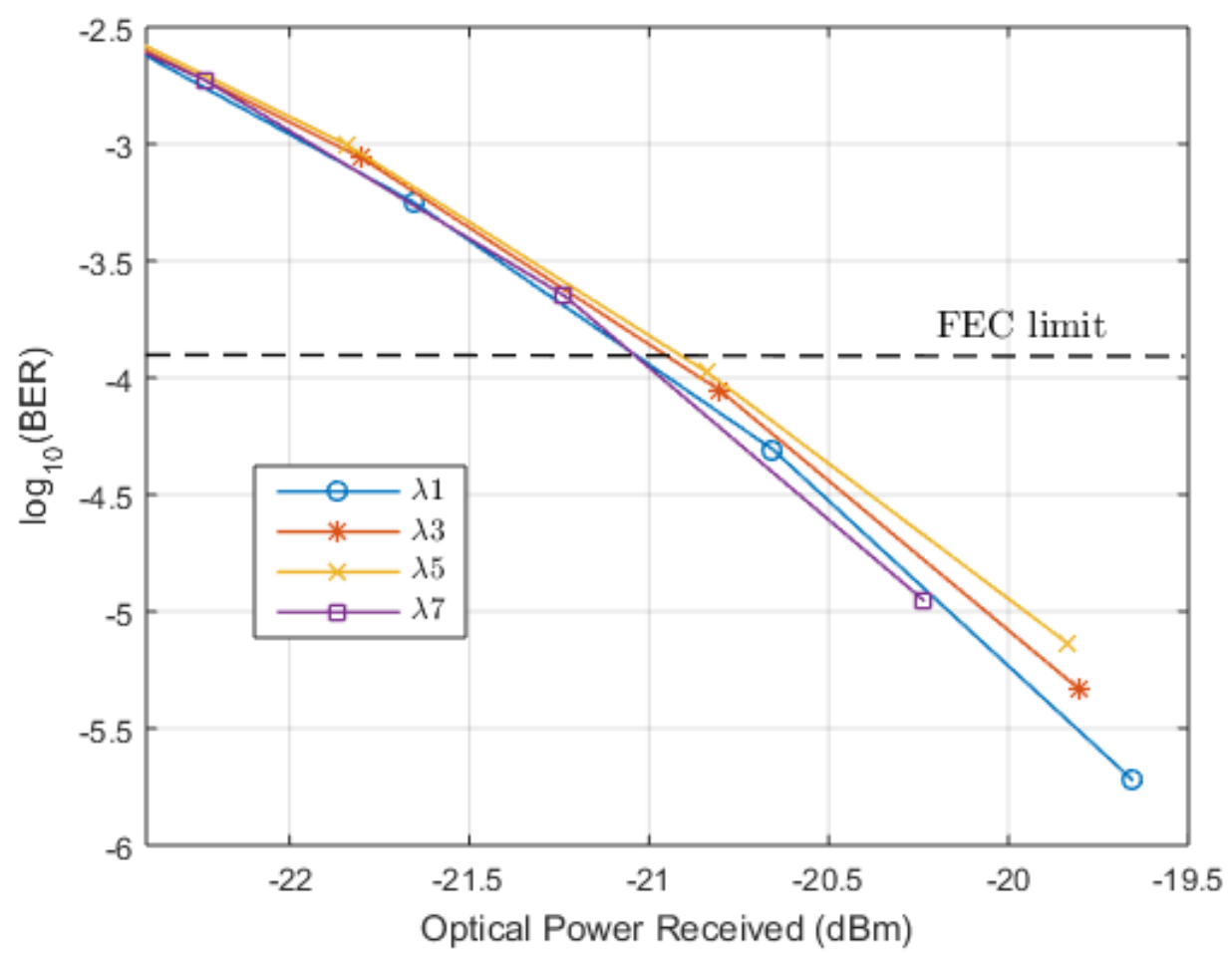

Fig. 7. BER versus optical power received for NRZ-OOK frequency domain interleaved system and $20 \mathrm{~km}$ SMF (Downstream).

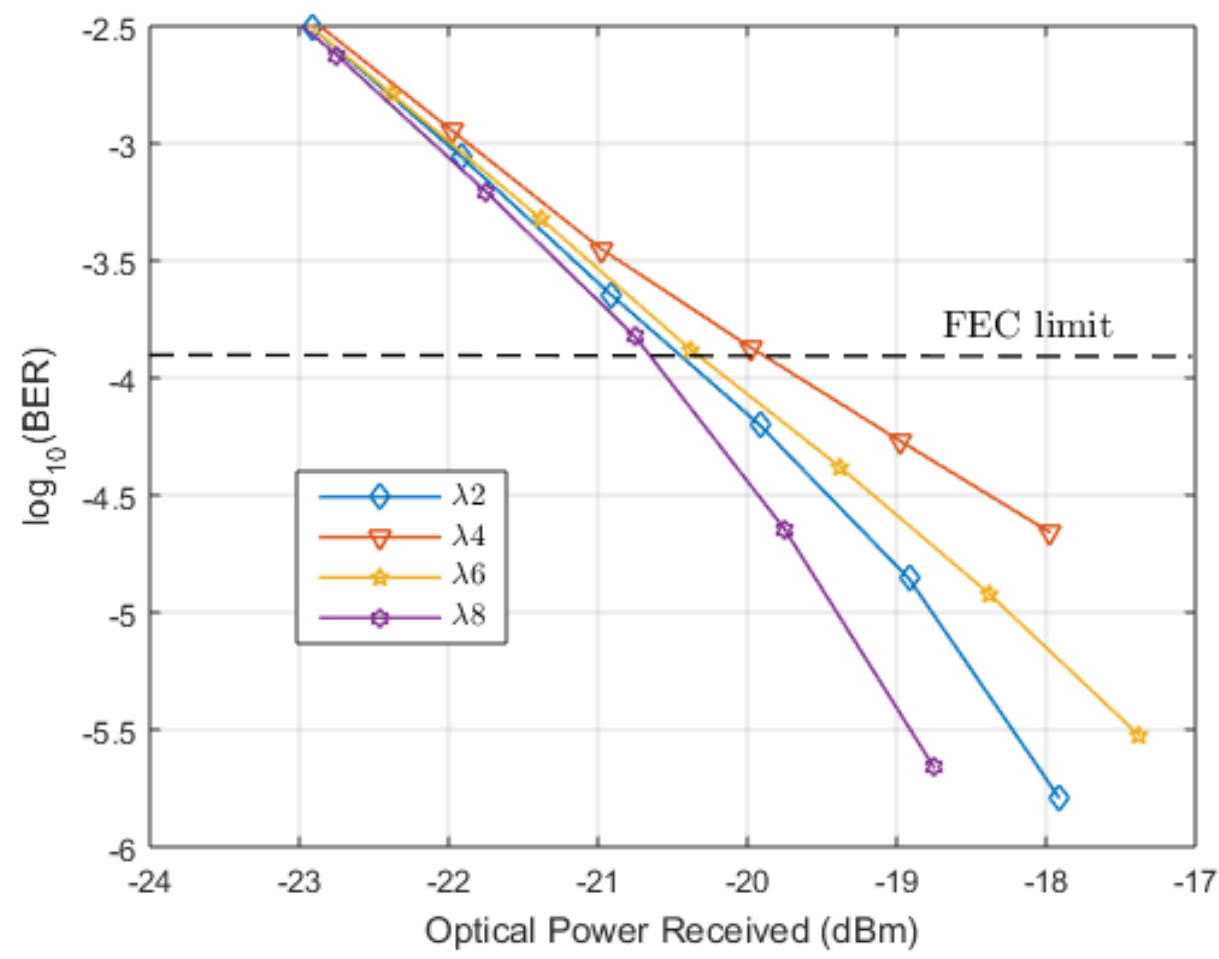

Fig. 8. BER versus optical power received for NRZ-OOK frequency domains interleaved system and $20 \mathrm{~km} \mathrm{SMF}$ (Upstream). 


\section{CONCLUSION}

In this paper, we explored the concept of frequency interleaving in passive optical networks based in Dense Wavelength Division Multiplexing (DWDM) to provide coexistence with previous generations of PONs in future generation of networks. The demultiplexing operation is introduced at the OLT and ONU side and is processed by a set of an all-optical passive element (Mach-Zehnder interferometer - MZI), which is a natural candidate to be implemented on a chip with silicon-oninsulator technology.

The RFS technique was chosen as the method for high OSNR quality optical wavelength generation due to its stability, flatness, flexibility on wavelength spacing control and low driving voltages.

The frequency interleaved system performance was evaluated in terms of BER as a function of several values optical received power for OOK modulation formats, after $20 \mathrm{~km}$ of SMF length, without any dispersion compensation for downstream and upstream path.

In the OOK system, we achieved $50 \mathrm{Gbits} / \mathrm{s}$ in both transmission paths and performance bellow the FEC limit for an optical power received higher than $-21 \mathrm{dBm}$ after $20 \mathrm{~km}$ of fiber length, for the downstream. For the upstream path, the performance remains under the FEC limit for received optical power higher than $-20 \mathrm{dBm}$.

\section{ACKNOWLEDGMENT}

The authors acknowledge the financial support received from Fundação de Amparo à Pesquisa e Inovação do Espírito Santo (FAPES) and Conselho Nacional de Desenvolvimento Científico e Tecnológico (CNPq).

\section{REFERENCES}

[1] S. Bindhaiq A. Sahmah, M. Supa'at, N. Zulkifli, A. B. Mohammad, R. Q. Shaddad, M. A. Elmagzoub and A. Faisal. "Recent development on time and wavelength-division multiplexed passive optical network (TWDM-PON) for nextgeneration passive optical network stage 2 (NG-PON2)," Optical Switching and Networking, vol. 15, pp. 53-56, January 2015, doi: 10.1016/j.osn.2014.06.007

[2] A. Cai, Z. Fan, K. Xu, M. Zukerman and C. Chan, "Elastic versus WDM networks with dedicated multicast protection," in IEEE/OSA Journal of Optical Communications and Networking, vol. 9, no. 11, pp. 921-933, Nov. 2017. doi: 10.1364/JOCN.9.000921

[3] Y. Sakaue, K. Taguchi, K. Hara, T. Shitaba, T. Taniguchi, S. Nishihara, K. Asaka, K. Suzuki and A. Otaka, "Demonstration of NG-PON2 Coexisting with Other Systems on Same ODN by Using WDM filter with Low Power Penalty of under $1.0 \mathrm{~dB}$," ECOC 2016; 42nd European Conference on Optical Communication, Dusseldorf, Germany, pp. 1-3, 2016.

[4] D. Nesset, "NG-PON2 Technology and Standards," in Journal of Lightwave Technology, vol. 33, no. 5, pp. 1136-1143, 1 March1, 2015. doi: 10.1109/JLT.2015.2389115.

[5] Coutinho, Bruno C., Lima, Fabio O., Lima Jr, Ivan T., Garcia, Anilton Salles and Segatto, Marcelo E. V. A Multilayer Approach for Optical Network Planning. Journal of Microwaves, Optoelectronics and Electromagnetic Applications, vol. 15, no.1, pp. 49-64, 2016. doi:10.1590/2179-10742016v15i1548

[6] H. Nejadriahi and V. J. Sorger, "On-chip Integrated All-Optical Fast Fourier Transform: Design and Analysis," in Frontiers in Optics 2017, OSA Technical Digest (online) (Optical Society of America, 2017), paper JW4A.46. 
[7] J. K. George, H. Nejadriahi and V. J. Sorger, "Towards On-Chip Optical FFTs for Convolutional Neural Networks," 2017 IEEE International Conference on Rebooting Computing (ICRC), Washington, DC, pp. 1-4, 2017. doi: 10.1109/ICRC.2017.8123675

[8] V. Torres-Company and A. M. Weiner, "Optical frequency comb technology for ultra-broadband radio-frequency photonics," Laser Photonics Rev. vol. 8, no. 3, pp. 368-393, 2014. doi: 10.1002/lpor.201300126

[9] V. Ataie, E. Temprana, L. Liu, E. Myslivets, B. P. Kuo, N. Alic and S. Radic, "Ultrahigh Count Coherent WDM Channels Transmission Using Optical Parametric Comb-Based Frequency Synthesizer," in Journal of Lightwave Technology, vol. 33, no. 3, pp. 694-699, 1 Feb.1, 2015. doi: 10.1109/JLT.2015.2388579

[10] T. Shao, R. Zhou, V. Vujicic, M. D. G. Pascual, P. M. Anandarajah and L. P. Barry, "100 km coherent Nyquist ultradense wavelength division multiplexed passive optical network using a tunable gain-switched comb source," in IEEE/OSA Journal of Optical Communications and Networking, vol. 8, no. 2, pp. 112-117, Feb. 2016. doi: $10.1364 / \mathrm{JOCN} .8 .000112$

[11] X. Zhou, X. Zheng, H. Wen, H. Zhang and B. Zhou, "Generation of broadband optical frequency comb with rectangular envelope using cascaded intensity and dual-parallel modulators," Opt. Commun., vol. 313, pp. 356-359, 2014.

[12] C. Lei, Y. Yu, M. Chen, H. Chen, S. Yang and S. Xie, "Temporally controlled wideband optical frequency comb generation based on recirculating frequency shifting," Microwave Photonics (MWP) and the 2014 9th Asia-Pacific Microwave Photonics Conference (APMP) 2014 International Topical Meeting on, Sendai, pp. 272-275. 2014. doi: 10.1109/MWP.2014.6994551

[13] D. Hillerkuss, T. Schellinger, M. Jordan, C. Weimann, F. Parmigiani, B. Resan, K. Weingarten, S. Ben-Ezra, B. Nebendahl, C. Koos, W. Freude and J. Leuthold, "High-Quality Optical Frequency Comb by Spectral Slicing of Spectra Broadened by SPM," in IEEE Photonics Journal, vol. 5, no. 5, pp. 7201011-7201011, Oct. 2013, Art no. 7201011. doi: 10.1109/JPHOT.2013.2280524

[14] R. Essiambre, G. Kramer, P. J. Winzer, G. J. Foschini and B. Goebel, "Capacity Limits of Optical Fiber Networks," in Journal of Lightwave Technology, vol. 28, no. 4, pp. 662-701, Feb.15, 2010. doi: 10.1109/JLT.2009.2039464

[15] R. J. L. Ferreira, D. M. Dourado, M. M. Rodrigues, M. L. Rocha, S. M. Rossi and D. M. Pataca, "All-optical fast fourier transform for processing an optical OFDM superchannel," 2017 SBMO/IEEE MTT-S International Microwave and Optoelectronics Conference (IMOC), Aguas de Lindoia, 2017, pp. 1-5.doi: 10.1109/IMOC.2017.8121075

[16] D. Hillerkuss, M. Winter, M. Teschke, A. Marculescu, J. Li, G. Sigurdsson, K. Worms, S. Ben Ezra, N. Narkiss, W. Freude, and J. Leuthold, "Simple all-optical FFT scheme enabling Tbit/s real-time signal processing," Opt. Express 18, pp. 9324-9340, 2010.

[17] Gerd Keiser, “Optical Fiber Communications”, $3^{\text {rd }}$ ed., MacGraw-Hill, New York, 2000, pp. 396-399.

[18] P. R. N. Marciano, D. V. N. Coelho, J. A. L. Silva, M. J. Pontes, and M. E. V. Segatto, "A New All-Optical OFDM Architecture for NG-PON2," in Latin America Optics and Photonics Conference, OSA Technical Digest (Optical Society of America, 2018), paper W3D.4. 